\title{
Therapeutic drug management: is it the future of multidrug-resistant tuberculosis treatment?
}

\author{
Shashikant Srivastava', Charles A. Peloquin², Giovanni Sotgiu ${ }^{3}$ and \\ Giovanni Battista Migliori ${ }^{4}$
}

\begin{abstract}
Affiliations: 'Dept of Medicine and Office of Global Health, UT Southwestern Medical Center, Dallas, TX, and ${ }^{2}$ College of Pharmacy and Emerging Pathogens Institute, University of Florida, Gainesville, FL, USA. ${ }^{3}$ Epidemiology and Medical Statistics Unit, Dept of Biomedical Sciences, University of Sassari-Research, Medical Education and Professional Development Unit, AOU Sassari, Sassari, and 4 WHO Collaborating Centre for TB and Lung Diseases, Fondazione S. Maugeri, Care and Research Institute, Tradate, Italy.
\end{abstract}

Correspondence: G.B. Migliori, S. Maugeri Foundation, Via Roncaccio 16, Tradate, Varese, 21049 Italy.

E-mail: giovannibattista.miglioriafsm.it

○

@ERSpublications

TDM can be helpful for multidrug-resistant tuberculosis therapeutic decisions. DBS can bypass logistical issues http://ow.ly/otsFp

Multidrug- and extensively drug-resistant (M/XDR) tuberculosis (TB) are emerging public health concerns $[1,2]$. In 2011, the World Health Organization (WHO) estimated 12 million prevalent cases of TB globally, which is equivalent to 170 cases per 100000 population, out of these an estimated 630000 cases were affected by MDR Mycobacterium tuberculosis strains [3]. Among the newly diagnosed patients $\sim 3.7 \%$ were infected by MDR-TB strains, but the worrisome fact is that the prevalence of MDR-TB among new cases in some Former Soviet Union countries exceeds $30 \%[4,5]$, XDR-TB has been identified in 84 countries and the average proportion of MDR-TB cases with an XDR-TB pattern is $9.0 \%$ [3]. Further adding to the problem are the reports of "totally drug resistant" TB [6, 7], a term currently not recognised by WHO [8, 9].

Treatment of drug resistant TB is more expensive and more toxic if compared with that prescribed for drugsusceptible TB, and currently takes up to 2 years of therapy [10]. The cost per patient to treat MDR-TB cases is incredibly high $[11,12]$ and, in spite of international public health efforts, the treatment outcome is not very promising [13-15]. DieL et al. [16] showed that direct treatment-related costs of MDR-TB patients can amount to $€ 52259$ in Germany (table 1).

In the largest MDR-TB cohort analysed to date [13] the proportion of cases treated successfully was $62 \%$, with $7 \%$ failing or relapsing, $9 \%$ dying and $17 \%$ defaulting; in the XDR-TB subgroup $40 \%$ achieved treatment success, $22 \%$ failed treatment or relapsed, whereas $15 \%$ died and $16 \%$ defaulted $[14,15]$.

In this issue of the European Respiratory Journal (ERJ) a Dutch group from Groningen [17] reported on the results of a prospective pharmacokinetic (PK) study aimed at quantifying the effect of clarithromycin on the exposure to linezolid. In simple terms they observed that clarithromycin, which has some activity against $\mathrm{TB}$ bacilli and is well tolerated, increases linezolid exposure (i.e. increases the blood levels of linezolid, which is a very expensive and toxic drug). The authors decided to quantify this phenomenon administering a fix dose of linezolid (300 mg twice a day) plus a variable one of clarithromycin (250-500 mg once a day). Using validated PK methods they demonstrated that linezolid exposure significantly increased after the coadministration of $500 \mathrm{mg}$ clarithromycin by a median (interquartile range) of 44\% (23-102\%), when

Received: April 262013 | Accepted: April 262013

Conflict of interest: None declared.

Copyright @ERS 2013 
TABLE 1 Direct costs of multidrug-resistant tuberculosis (MDR-TB) therapy in a European low-income country

\#: data refer to adult cases only. Data from [16].

compared with baseline conditions, whereas $250 \mathrm{mg}$ clarithromycin had no statistically significant effect. Co-administration was well tolerated by most patients; no patients experienced severe adverse events.

The clinical implications of these findings are as follows: 1) clarithromycin might be used as a booster for linezolid, exactly as low-dose ritonavir is used to increase protease inhibitor exposure in combined antiretroviral therapy; and 2) the relatively cheap clarithromycin could reduce the prescribed dose of the expensive linezolid while the same exposure is maintained (and the same toxicity is expected), making more economic resources available to treat other (more) MDR-TB cases.

Also in this edition of the ERJ, a letter by the same Dutch group provides an example of the clinical applications of therapeutic drug monitoring (TDM) [18]. The study provides, for the first time, evidence of anti-TB drugs' penetration into a human tissue (a TB-destroyed lung in this case) in parallel to their blood concentration. The case described in their article is complicated and is of a young Somalian female where the M. tuberculosis strain is resistant to all first-line drugs as well as a WHO group V one (prothionamide).

TDM was performed, as routinely undertaken in this clinic, for the core drugs prescribed including linezolid [19] and moxifloxacin. The study results show that penetration in the lung was excellent, based upon tissue homogenates, in both the more and less destroyed segments of the lung.

This study provides for the first time two important pieces of clinical evidence: 1) the feasibility of measuring in parallel, through TDM, blood and lung tissue concentrations of anti-TB drugs and 2) linezolid's and moxifloxacin's excellent penetration even in the destroyed lung tissue.

TDM, although not yet popular among TB specialists, is well known in clinical pharmacology [20-22]. It is based on the collection of blood samples and allows the ex vivo evaluation of blood-drug concentration, and, consequently, of its potential quantitative effect on the pharmacological target. Development of drug resistance in $M$. tuberculosis is attributed to inadequate treatment [23, 24], particularly inadequate dose or dosing frequency, non-adherence to the prescribed regimen, and PK variability [25, 26]. While new technologies/assays have been developed for the rapid detection of drug resistance [27] TDM has not been used to its full potential for the management of TB therapy [22], where a drug's low serum level is readily corrected with dose adjustment.

Table 2 summarises the PK parameters of the second-line anti-TB drugs. Such adjustments are important when treating patients who are slow to respond to treatment (i.e. malabsorption, inaccurate dosing, altered metabolism, drug-drug interactions [13,21], or PK variability [25]), have drug-resistant TB, are at risk of drug-drug interactions, have adverse events or intolerance to a given drug when this drug is essential to ensure a positive outcome or have concurrent disease states that significantly complicate the pharmacological metabolism. Such patients may benefit from TDM precluding the development of further drug resistance because of mycobacterial exposure to sub-inhibitory drug concentrations. In the more complicated situation (e.g. XDR-TB strain) where only four to five effective drugs are available, TDM can even be life-saving if it detects and corrects malabsorption before further resistance is selected.

While TDM seems a promising approach, logistical and cost-related problems are still limiting its application to research-oriented institutions. An alternative cost-effective approach is "dried blood spot" (DBS) [29] (fig. 1), which makes TDM a much easier technique to perform, even in remote settings. DBS compared with conventional venous blood sampling, which requires hospitalisation, has the advantages of easier sampling, smaller blood volume usage, storage and transportation. DBS is in its infancy and requires additional considerations for development of drug analysis methods and their validation.

The cost of TDM varies from laboratory to laboratory and from country to country. In one study the cost has been calculated to be US\$80 per individual drug [20], whereas others charge US\$80 for a single test of a 
TABLE 2 Pharmacokinetic parameters of the second line anti-tuberculosis drugs [28]

\begin{tabular}{|c|c|c|c|c|c|}
\hline Drug & Dose & $\mathrm{C}_{\max } \mu \mathrm{g} \cdot \mathrm{mL}^{-1}$ & Time to $C_{\max } \mathrm{h}$ & $t_{1 / 2} h$ & AUC $0-24 \mu \mathrm{g} \cdot \mathrm{h}^{-1} \cdot \mathrm{mL}^{1}$ \\
\hline Linezolid & $600 \mathrm{mg}$ & 20.4 & 1.4 & 5.8 & 140.8 \\
\hline Clarithromycin & $500 \mathrm{mg}$ & $1-1.5$ & $2-4$ & $3-7$ & \\
\hline Clofazimine & $100 \mathrm{mg}$ & $05-2$ & $2-7$ & 10 days -2 weeks & 1.5 \\
\hline Ofloxacin & $800 \mathrm{mg}$ & 10.5 & 1 & 7 & 103 \\
\hline Amikacin & $15 \mathrm{mg} \cdot \mathrm{kg}^{-1}$ i.v. & 46 & & 2.5 & \\
\hline Kanamycin & $15 \mathrm{mg} \cdot \mathrm{kg}^{-1}$ i.v. & 44 & & 2.2 & \\
\hline Capreomycin & $1 \mathrm{~g}$ i.m. & 32 & & & \\
\hline Ethionamide & $500 \mathrm{mg}$ & 1.35 & 2 & 1.63 & 2.8 \\
\hline Prothionamide & $250 \mathrm{mg}$ & 2.5 & 3.4 & 3 & 11.3 \\
\hline Cycloserine & $250 \mathrm{mg}$ & $8-9$ & $2-3$ & 25.1 & \\
\hline
\end{tabular}

$t_{1 / 2}$ : half-life; AUC: area under the curve from 0 to $24 \mathrm{~h}$.

single drug, and US\$70 per test if two or more time points are tested, per patient (personal communication; Charles A. Peloquin, College of Pharmacy and Emerging Pathogens Institute, University of Florida, Gainesville, FL, USA). In other words, the total cost to test four drugs at 2 and $6 \mathrm{~h}$ post observed oral doses will add to $8 \times \$ 70$ or $\$ 560$, and this does not include the cost of collecting, processing, and shipping the samples. Although this seems to add a significant financial burden, if one has to consider the total cost of MDR-TB treatment/patient (including the cost of adverse events' management, the cost of consequences of unsuccessful outcomes (i.e. failure/death/relapse) and drug-resistant M. tuberculosis transmission to close contacts), early intervention to adjust the dose and potentially shorten the duration of therapy, TDM will likely prove cost-effective given the very high overall cost to treat M/XDR-TB $[11,12,16]$.

Unfortunately, there are not many established laboratories offering TDM and interpretation of the results. However, the scenario is changing, in the USA some laboratories are starting to do drug testing in-house, with one lab (University of Florida, Florida, FL, USA) offering both the assays and their individualised interpretation (personal communication; Charles A. Peloquin and [20]). Whereas in Europe the group of scientists in Nijmegen (the Netherlands) has begun to set up a proficiency testing programme (personal communication; Charles A. Peloquin). Thus the number of international laboratories offering TDM should grow in the near future.

In perspective, we can dream of a future quality assurance system designed in a similar way as that developed by WHO and the International Union Against Tuberculosis and Lung Disease (IUATLD) for first-line anti-TB drugs drug-susceptibility testing (the scheme for second-line drugs being presently under development) [3, 30]. A few, quality-controlled, reference laboratories might receive DBS from reference MDR-TB clinics (fig. 1). Although, as of today, TDM is perceived as a technique applicable to high income

FIGURE 1 Dried blood spot (DBS) for therapeutic drug monitoring. Blood is collected on paper strips (a) and packed in a plastic bag with a desiccant to keep the strip dry. Samples can then be transported (b) via regular post or any other suitable means. The DBS is collected from the strip and the drug is extracted and concentrations subsequently measured using validated methods. Personal communication D.H. Vu (University of Groningen, Groningen, the Netherlands, and Hanoi University of Pharmacy, Hanoi, Vietnam).

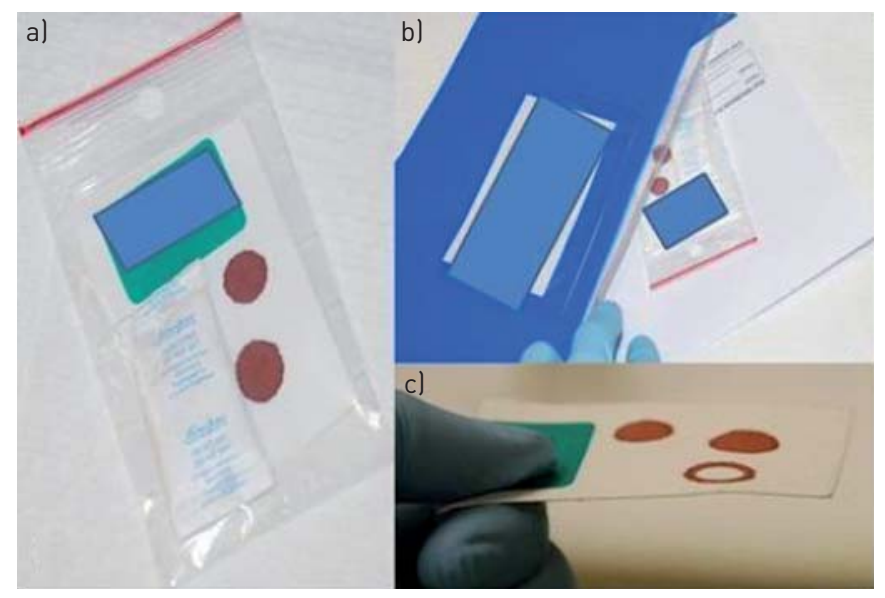


countries only, a few arguments might open the way for future expansion in its use. 1) Although TDM, as discussed earlier, is a relatively expensive examination, the savings in the amount of anti-TB drugs prescribed and the (costs related to the) reduction of adverse events will be able to self-pay for it cost. 2) DBS is simple to collect, this operation can be done in any peripheral health clinic if needed. 3) DBS does not contain any infectious material and can be sent via normal mail, whereas shipment of $M$. tuberculosis strains should be done according to precise international regulations and is very expensive.

Last but not least, no existing guideline presently recommends the use of TDM in difficult-to-treat MDRTB cases [10, 31, 32]. We hope that the evidence provided in these ERJ articles, together with the much needed evidence that will (hopefully) be available soon that will allow this technique to be included, sooner or later, among those "officially" endorsed to better manage the most severe M/XDR-TB cases.

While all of the suggestions discussed here are pharmacologically sound, and supported by anecdotal clinical data, it remains unlikely that definitive proof from prospective, randomised clinical trials will ever be forthcoming. Thus, as of today, it remains a clinical decision to perform TDM, very similar to the decision to order complete blood counts, computerised tomography scans, or magnetic resonance scans in TB patients. All of these tests, including TDM, are useful if they inform treatment decisions. With TDM, the decision is what dose and frequency to use for a given drug. When only four or five effective drugs are available to treat a complicated M/XDR-TB case, and losing a single drug might represent the patient's death sentence, any effort should be done to use these "life-saving" drugs in the best possible way.

\section{References}

Raviglione M, Marais B, Floyd K, et al. Scaling up interventions to achieve global tuberculosis control: progress and new developments. Lancet 2012; 379: 1902-1913.

2 Zumla A, Kim P, Maeurer M, et al. Zero deaths from tuberculosis: progress, reality, and hope. Lancet Infect Dis 2013; 13: 285-287.

World Health Organization. Global tuberculosis report 2012. Geneva, World Health Organization, 2012.

Skrahina A, Hurevich H, Zalutskaya A, et al. Alarming levels of drug-resistant tuberculosis in Belarus: results of a survey in Minsk. Eur Respir J 2012; 39: 1425-1431.

5 Migliori GB, Dara M, De Colombani P, et al. Multidrug-resistant tuberculosis in Eastern Europe: still on the increase? Eur Respir J 2012; 39: 1290-1291.

6 Migliori GB, De Iaco G, Besozzi G, et al. First tuberculosis cases in Italy resistant to all tested drugs. Euro Surveill 2007; 12: E070517.1.

$7 \quad$ Udwadia Z, Vendoti D. Totally drug-resistant tuberculosis (TDR-TB) in India: every dark cloud has a silver lining. J Epidemiol Community Health 2013; 67: 471-472.

8 Migliori GB, Centis R, D’Ambrosio L, et al. Totally drug-resistant and extremely drug-resistant tuberculosis: the same disease? Clin Infect Dis 2012; 54: 1379-1380.

9 World Health Organization. Drug-resistant tuberculosis. Frequently asked questions, 2012 www.who.int/tb/ challenges/mdr/tdrfaqs/en/index.html Date last accessed: Apr 22, 2013. Date last updated: January 2012.

10 Falzon D, Jaramillo E, Schünemann HJ, et al. WHO guidelines for the programmatic management of drug-resistant tuberculosis: 2011 update. Eur Respir J 2011; 38: 516-528.

11 Floyd K, Hutubessy R, Kliiman K, et al. Cost and cost-effectiveness of multidrug-resistant tuberculosis treatment in Estonia and Russia. Eur Respir J 2012; 40: 133-142.

12 Loddenkemper R, Sotgiu G, Mitnick CD. Cost of tuberculosis in the era of multidrug resistance: will it become unaffordable? Eur Respir J 2012; 40: 9-11.

13 Ahuja SD, Ashkin D, Avendano M, et al. Multidrug resistant pulmonary tuberculosis treatment regimens and patient outcomes: an individual patient data meta-analysis of 9,153 patients. PLoS Med 2012; 9: e1001300.

14 Falzon D, Gandhi N, Migliori GB, et al. Resistance to fluoroquinolones and second-line injectable drugs: impact on multidrug-resistant TB outcomes. Eur Respir J 2013; 42: 156-168.

15 Migliori GB, Sotgiu G, Gandhi NR, et al. Drug resistance beyond extensively drug-resistant tuberculosis: individual patient data meta-analysis. Eur Respir J 2013; 42: 169-179.

16 Diel R, Rutz S, Castell S, et al. Tuberculosis: cost of illness in Germany. Eur Respir J 2012; 40: 143-151.

17 Bolhuis MS, van Altena R, van Soolingen D, et al. Clarithromycin increases linezolid exposure in multidrugresistant tuberculosis patients. Eur Respir J 2013; 42: 1614-1621.

18 Akkerman OW, van Altena R, Klinkenberg T, et al. Drug concentration in lung tissue in multidrug-resistant tuberculosis. Eur Respir J 2013; 42: 1750-1752.

19 Sotgiu G, Centis R, D'Ambrosio L, et al. Efficacy, safety and tolerability of linezolid containing regimens in treating MDR-TB and XDR-TB: systematic review and meta-analysis. Eur Respir J 2012; 40: 1430-1442.

20 Heysell SK, Moore JL, Keller SJ, et al. Therapeutic drug monitoring for slow response to tuberculosis treatment in a state control program, Virginia, USA. Emerging Infect Dis 2010; 16: 1546-153.

Peloquin CA. Pharmacological issues in the treatment of tuberculosis. Ann N Y Acad Sci 2001; 953: 157-164. Peloquin CA. Therapeutic drug monitoring in the treatment of tuberculosis. Drugs 2002; 62: 2169-2183.

Migliori GB, Sotgiu G, D’Ambrosio L, et al. TB and MDR/XDR-TB in European Union and European Economic Area countries: managed or mismanaged? Eur Respir J 2012; 39: 619-625.

24 Raviglione MC, Lange C, Migliori GB. Preventing and managing antimicrobial resistance: imperative for chest physicians. Eur Respir J 2011; 37: 978-981.

25 Srivastava S, Pasipanodya JG, Meek C, et al. Multidrug-resistant tuberculosis not due to noncompliance but to between-patient pharmacokinetic variability. J Infect Dis 2011; 204: 1951-1959.

26 Van der Werf MJ, Langendam MW, Huitric E, et al. Multidrug resistance after inappropriate tuberculosis treatment: a meta-analysis. Eur Respir J 2012; 39: 1511-1519. 
27 Tortoli E, Russo C, Piersimoni C, et al. Clinical validation of Xpert MTB/RIF for the diagnosis of extrapulmonary tuberculosis. Eur Respir J 2012; 40: 442-447.

28 Garcia-Prats A, Donald P, Hesseling A, et al. Second-Line Antituberculosis Drugs in Children: A Commissioned Review for the World Health Organization 19th Expert Committee on the Selection and Use of Essential Medicines. www.who.int/selection_medicines/committees/expert/19/applications/TB_624_C_R.pdf Date last accessed: January 2013. Date last updated: January 2013.

29 Vu DH, Alffenaar JWC, Edelbroek PM, et al. Dried blood spots: a new tool for tuberculosis treatment optimization. Curr Pharm Des 2011; 17: 2931-2939.

30 Fattorini L, Migliori GB, Cassone A, et al. Proficiency testing of first- and second-line anti-tuberculosis drugs in Italy. Eur Respir J 2012; 39: 1263-1266.

31 Tuberculosis Coalition for Technical Assistance. International Standards for Tuberculosis Care (ISTC), 2nd Edn. The Hague, Tuberculosis Coalition for Technical Assistance, 2009.

32 Migliori GB, Zellweger JP, Abubakar I, et al. European union standards for tuberculosis care. Eur Respir J 2012; 39: 807-819. 